\title{
Positioning control system with high-speed starting and stopping for a DC motor using bang-bang control
}

\author{
Hiroki Shibasaki ${ }^{1,}$, Takehito Fujio ${ }^{1}$, Ryo Tanaka ${ }^{1}$, Hiromitsu Ogawa ${ }^{1}$, Yoshihisa Ishida ${ }^{1,2}$ \\ ${ }^{1}$ Graduate School of Science and Technology, Meiji University, Kawasaki, JAPAN \\ ${ }^{2}$ School of Science and Technology, Meiji University, Kawasaki, JAPAN

\section{Email address:} \\ shiba@meiji.ac.jp (H. Shibasaki), ce41087@meiji.ac.jp (T. Fujio), rtanaka@meiji.ac.jp (R. Tanaka), h_ogawa@meiji.ac.jp (H. Ogawa), \\ ishida@isc.meiji.ac.jp (Y. Ishida)
}

\section{To cite this article:}

Hiroki Shibasaki, Takehito Fujio, Ryo Tanaka, Hiromitsu Ogawa, Yoshihisa Ishida. Positioning Control System with High-Speed Starting and Stopping for a DC Motor Using Bang-Bang Control. Automation, Control and Intelligent Systems. Vol. 2, No. 4, 2014 , pp. $62-70$. doi: 10.11648/j.acis.20140204.13

\begin{abstract}
This paper proposes a positioning control system with high-speed starting and stopping for a DC motor using bang-bang control. The control system of the proposed method continuously operates from the bang-bang control to proportional-integral-derivative (PID) control. The bang-bang control controls the equipment for high-speed starting and stopping. However, torque disturbances in the equipment may prevent the target-value response from stopping at a precise point. Therefore, we introduce a switching control system, which operates on the basis of the velocity signal. The proposed system includes various design factors, such as calculation of the switching time of bang-bang input and a method for continuous operation from the bang-bang control to PID control. Theoretical analysis describes the design details of the proposed method. Simulation and experimental studies show the results of various cases to indicate the effectiveness of the proposed method.
\end{abstract}

Keywords: Bang-Bang Control, PID Control, Switching Control, Disturbance Observer

\section{Introduction}

In industry, proportional-integral-derivative (PID) control [1] [2] is used to control the actuator. Furthermore, it is applied to design methods in a wide range of processes in the areas from research and development to production lines. Although an advantage of the PID controller is its general ease of design, the PID controller also has numerous limitations. For instance, tuning of PID controller is determined by the rule of experience. Otherwise, it is difficult to reduce the influence of overshoot. Furthermore, industrial demands require precise starting and stopping in high speed conditions of the actuator.

Bang-bang controllers [3] control the actuator for rapid movement and stopping; This means that it inputs maximum or minimum values to the actuator in open loop, which is known as "Bang-Bang input".

Research on bang-bang control has been conducted worldwide. Lasalle [4] reported the principle of the bang-bang control. K. Nagakura [5] described time-optimal precise positioning control using a de servomotor. Rillings. et al. [6] proposed a bang-bang control strategy that uses a tabular adaptive model in a nonlinear system. In addition, K. Furuta et al. [7] designed a bang-bang position control that uses VSS control for the directive drive motor. N. Koreta et al. [8] conducted a study of high accuracy of machine tools with bang-bang control. Further, M. G.Vasek et al. [9] proposed bang-bang control for the double integrator plant in positioning control. Y. Yoshida et al. [10] proposed bang-bang control of a crane in real time. Authors [11] proposed the high speed activation and stopping control system using bang-bang control and simulated for a DC motor.

The present study proposes a position control with high-speed starting and stopping for a DC motor that use bang-bang control. It gradually and continuously converts, a control system started by bang-bang control to PID control. When the system converts, the system generally becomes discontinuous. Therefore, the initial value of the integrator in PID control, which is determined by the whole system is introduced in the control system. In addition, we introduce a disturbance observer for overcoming the variation in torque disturbance. We present the theoretical 
analysis and design method in detail We show the simulation results and also confirm the responses by using the real system, the DC motor [12].

This paper is constituted as follows. Section 2 shows the DC motor and Section 3 describes the bang-bang control. Section 4 proposes our method and gives design details and theoretical analysis. Section 5 shows the simulation results in various conditions, and Section 6 discusses the experimental study using the DC motor. Finally, we state the conclusion of this paper in Section 7.

\section{DC Motor}

This section describes the DC motor construction [5]. DC motor electrical characteristic and mechanical characteristic are written as follows:

$$
\left\{\begin{array}{l}
L \frac{d i(t)}{d t}+R i(t)+K_{E} \omega(t)=u(t) \\
J \frac{d \omega(t)}{d t}+D \omega(t)+T_{L} \operatorname{sgn}\{\omega(t)\}=K_{T} i(t)
\end{array}\right.
$$

which includes the consists of following parameters.

$$
\begin{array}{ll}
L & \text { inductance of circuit (Henry) } \\
R & \text { resistance of circuit }(\Omega) \\
K_{E} & \text { voltage constant of motor }(\mathrm{V} / \mathrm{rps}) \\
K_{T} & \text { torque constant of motor }(\mathrm{g} \cdot \mathrm{cm} / \mathrm{A}) \\
J & \text { inertia of the rotating part }\left(\mathrm{g} \cdot \mathrm{cms}^{2}\right) \\
D & \text { viscous friction coefficient }(\mathrm{g} \cdot \mathrm{cm} / \mathrm{rps}) \\
T_{L} & \text { solid friction }(\mathrm{g} \cdot \mathrm{cm}) \\
i & \text { current }(\mathrm{A}) \\
u(t) & \text { input }(\mathrm{V}) \\
\omega(t) & \text { velocity }(\mathrm{rad} / \mathrm{s})
\end{array}
$$

(1) is rewritten in the Laplace transform as follows:

$$
\left\{\begin{array}{l}
L s I(s)+R I(s)+K_{E} \Omega(s)=U(s) \\
J_{S} \Omega(s)+D \Omega(s)+T_{L} \Omega(s)=K_{T} I(s)
\end{array}\right.
$$

In this equation, the inductance of circuit $L$ is negligible, because it is quite small. From (2), the velocity and the position are as follows:

$$
\left\{\begin{array}{l}
\Omega(s)=\frac{T_{m}}{T_{m} s+1}\left(\frac{K_{T}}{J R} U(s)-\frac{T_{L}}{J}\right) \\
\theta(s)=\frac{T_{m}}{s\left(T_{m} s+1\right)}\left(\frac{K_{T}}{J R} U(s)-\frac{T_{L}}{J}\right)
\end{array}\right.
$$

$$
T_{m}=\frac{J R}{R D+K_{T} K_{E}} .
$$

\section{Bang-Bang Control}

Fig. 1 shows a block diagram of the bang-bang control and Fig.2 shows an example response. The maximum input to the DC motor produces the maximum speed. Therefore, the bang-bang control is the control system to stop to the target-value in the minimum time. However, when the plant has load changes or disturbances, controlling a plant using this method is difficult.

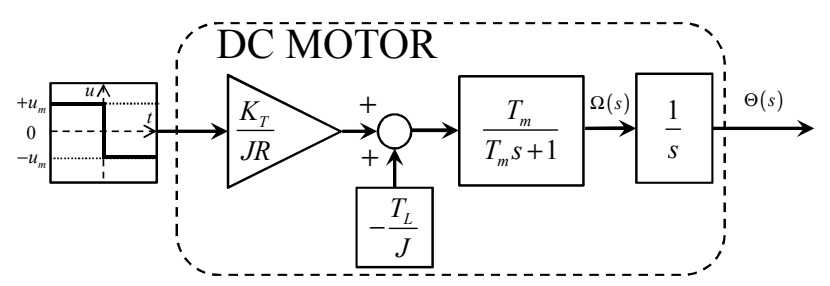

Figure 1. Block diagram of bang-bang control
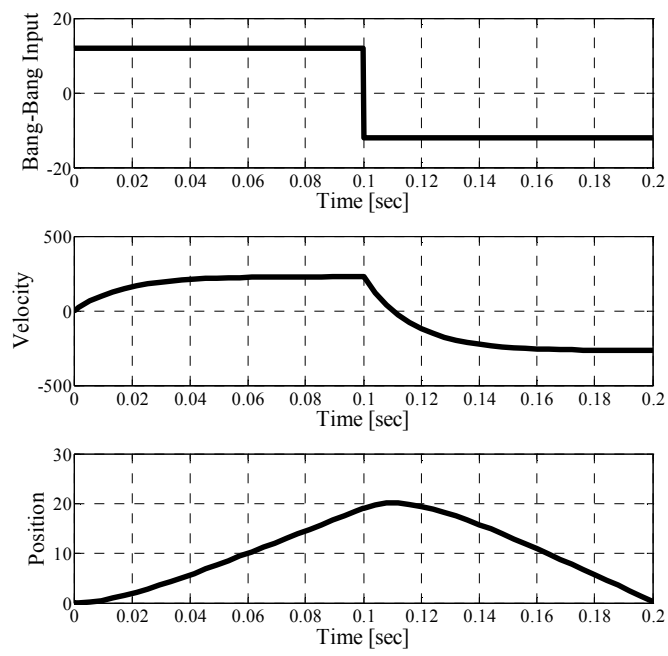

Figure 2. Example responses of bang-bang Control

\section{Proposed Method}

In the proposed method, the motor position is moved near the target value by the bang-bang control and is then converted to PID control. Fig. 3 shows a block diagram of the proposed method.

In this section, we discuss the design of the control system and offer a theoretical case. Fig. 4 graphically shows our proposed method. 


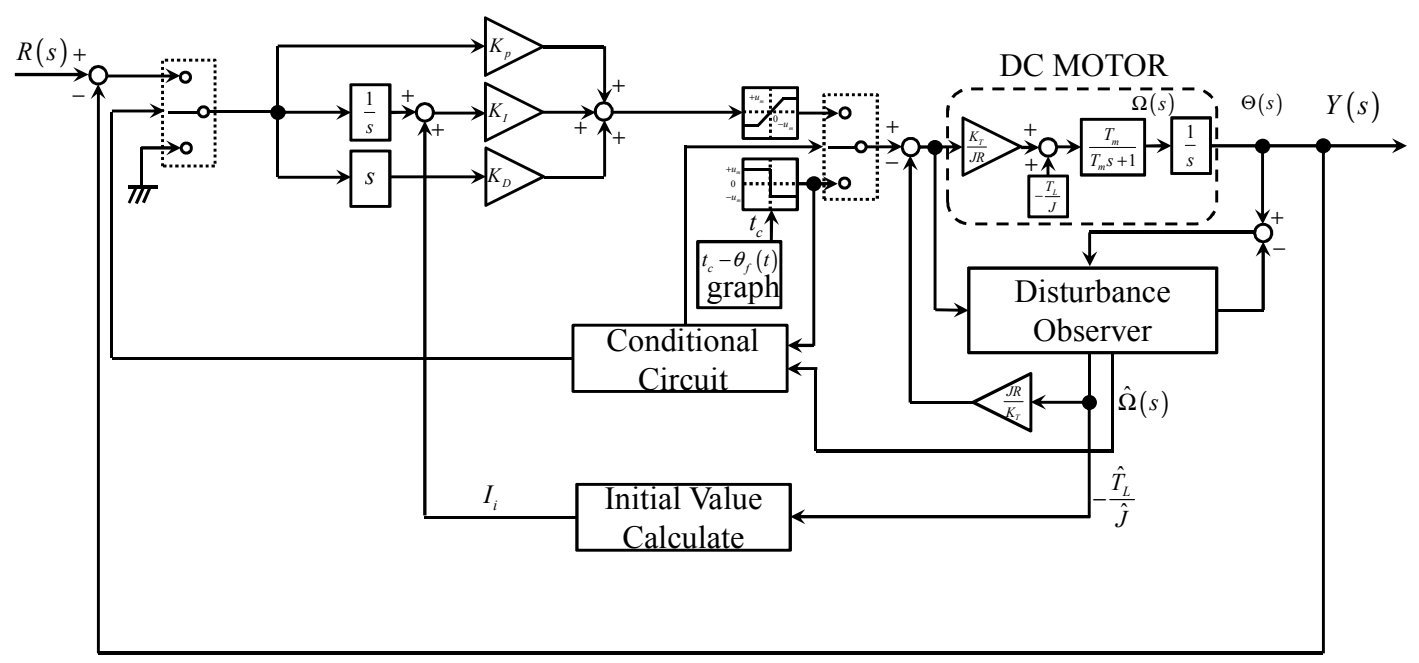

Figure 3. Block diagram of proposed method

\subsection{Design of the Control System}

First, we build the graph, the relationship of the position switching signal $\theta_{f}(t)$ and the switching time $t_{c}$ in bang-bang input using the velocity switching signal $\omega_{f}(t)$. $\theta_{f}(t)$ is measured using the bang-bang control as shown in Fig.1. Then, the data are arranged using the least squares method after measurement, where, $t_{\mathrm{c}}$ and $\omega_{f}(t)$ are arbitrarily determined, e.g., $t_{\mathrm{c}}=0.01$, or $0.1 ; \omega_{f}(t)=100$ or 200 .

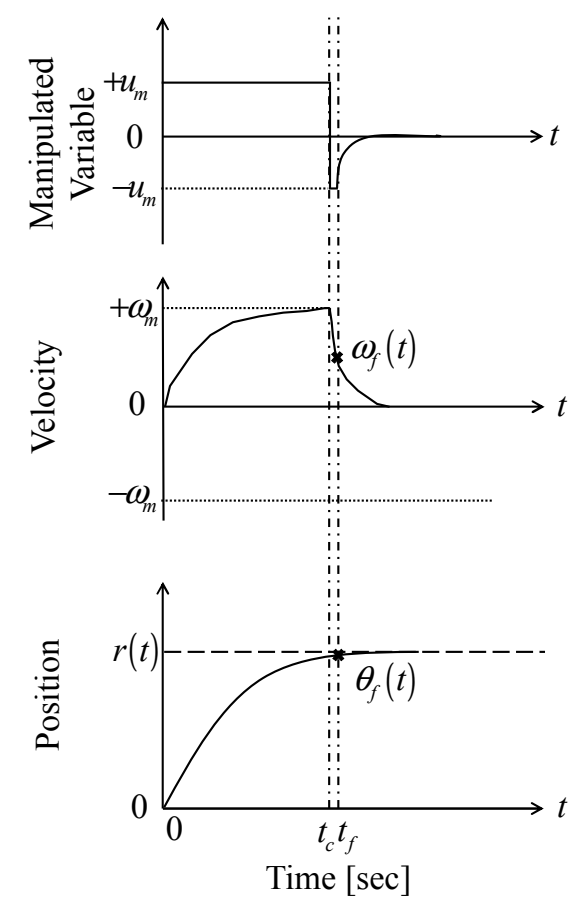

Figure 4. Explanation responses of proposed method

Second, we determine the target-value $r(t)$ and obtain the position switching signal $\theta_{f}(t)$. From the $t_{c}-\theta_{f}(t)$ graph, we get the switching time $t_{c}$ in the bang-bang input.
Finally, we design PID control using the pole placement method. The system is converted from bang-bang control to PID control on the basis of the $t_{c^{-}} \theta_{f}(t)$ graph.

\subsection{Theoretical of the Control System}

This section describes to derive switching time $t_{c}$ in bang-bang input and convert time $t_{f}$ to PID control.

The differential equation about the velocity is written as follows:

$$
\frac{d \omega(t)}{d t}+\frac{\omega(t)}{T_{m}}=-\left(\frac{1}{T_{m}}-\frac{D}{J}\right) \omega_{m}-\frac{T_{L}}{J}, \quad \omega_{m}=\frac{u_{m}}{K_{E}},
$$

where, $\omega_{m}$ is max velocity

A. Acceleration Time $\left(u=u_{m}\right)$

The velocity $\omega(t)$ in the acceleration time is as follows:

$$
\begin{aligned}
\omega(t) & =T_{m}\left\{\left(\frac{1}{T_{m}}-\frac{D}{J}\right) \omega_{m}-\frac{T_{L}}{J}\right\}\left(1-e^{-\frac{t}{T_{m}}}\right) \\
& =T_{m}\left\{\frac{K_{T}}{J R} u_{m}-\frac{T_{L}}{J}\right\}\left(1-e^{-\frac{t}{T_{m}}}\right)
\end{aligned}
$$

B. Deceleration Time $\left(u=-u_{m}\right)$

Velocity $\omega(t)$ in deceleration time is follows:

$$
\begin{aligned}
\omega(t) & =-T_{m}\left\{\left(\frac{1}{T_{m}}-\frac{D}{J}\right) \omega_{m}+\frac{T_{L}}{J}\right\}\left(1-e^{-\frac{t}{T_{m}}}\right) \\
& =-T_{m}\left\{2 \frac{K_{T}}{J R} u_{m}+\frac{T_{L}}{J}\right\}\left(1-e^{-\frac{t}{T_{m}}}\right)
\end{aligned}
$$

From $A$ ) and $B$ ), the velocity switching signal $\omega_{f}(t)$ and the position switching signal $\theta_{f}(t)$ are as follows: 


$$
\left\{\begin{aligned}
& \omega_{f}(t)=\omega\left(t_{f}\right)=T_{m}\left\{\frac{K_{T}}{J R} u_{m}-\frac{T_{L}}{J}\right\}\left(1-e^{-\frac{t_{c}}{T_{m}}}\right) \\
&-T_{m}\left\{2 \frac{K_{T}}{J R} u_{m}+\frac{T_{L}}{J}\right\}\left(1-e^{-\frac{t_{f}-t_{c}}{T_{m}}}\right) \\
& \theta_{f}(t)=\theta\left(t_{f}\right) \\
&=T_{m}\left\{\frac{K_{T}}{J R} u_{m}-\frac{T_{L}}{J}\right\}\left[t_{c}-T_{m}\left(1-e^{-\frac{t_{c}}{T_{m}}}\right)\right] \\
& \cdots+T_{m}\left\{\frac{K_{T}}{J R} u_{m}-\frac{T_{L}}{J}\right\}\left(1-e^{-\frac{t_{c}}{T_{m}}}\right)\left(t_{f}-t_{c}\right) \\
& \cdots-T_{m}\left\{2 \frac{K_{T}}{J R} u_{m}+\frac{T_{L}}{J}\right\}\left[\left(t_{f}-t_{c}\right)-T_{m}\left(1-e^{-\frac{t_{f}-t_{c}}{T_{m}}}\right)\right]
\end{aligned}\right.
$$

$\omega_{f}(t)$ and $\theta_{f}(t)$ are already specified. By solving the simultaneous equations in (8), the switching time $t_{c}$ and $t_{f}$ can be calculated.

Second, we consider the design of the entire system and the method for designing the PID controller.

From Fig. 3, the target-value response is as follows:

$$
Y(s)=\frac{K_{d} K_{T} T_{m} s^{2}+K_{p} K_{T} T_{m} s+K_{i} K_{T} T_{m}}{J R T_{m} s^{3}+\left(J R+K_{d} K_{T} T_{m}\right) s^{2}+K_{p} K_{T} T_{m} s+K_{i} K_{T} T_{m}} R(s)
$$

From above equation, we can design PID controller by the pole placement method.

Here, a reference model is determined as follows:

$$
\frac{\alpha^{3}}{(s+\alpha)^{3}}=\frac{\alpha^{3}}{s^{3}+3 \alpha s^{2}+3 \alpha^{2} s+\alpha^{3}},
$$

where, $\alpha$ is the model parameter.

The denominator of the target-value response can be compared with denominator of the reference model. Therefore, PID controller gains $K_{p}, K_{i}$, and $K_{d}$ are written as follows:

$$
\left\{\begin{array} { l } 
{ \alpha ^ { 3 } = \frac { K _ { i } K _ { T } } { J R } } \\
{ 3 \alpha ^ { 2 } = \frac { K _ { p } K _ { T } } { J R } } \\
{ 3 \alpha = \frac { 1 } { T _ { m } } + \frac { K _ { d } K _ { T } } { J R } }
\end{array} \Rightarrow \left\{\begin{array}{l}
K_{p}=3 \alpha^{2} \frac{J R}{K_{T}} \\
K_{i}=\alpha^{3} \frac{J R}{K_{T}} \\
K_{d}=\left(3 \alpha-\frac{1}{T_{m}}\right) \frac{J R}{K_{T}}
\end{array}\right.\right.
$$

Finally, the system can be converted from bang-bang control to PID control. However, it must maintain continuousness during the conversion process. Therefore, we focus on the integrator of the PID controller and we determine its initial value.

Fig. 5 shows a block diagram of the proposed method used to introduce the initial value of the integrator.

First, the transfer function is derived from each signal $R(s), I_{i},-T_{L} / J, I_{v}$, and $I_{p}$, to velocity $Y_{v}(s)$.

Then, the characteristic of equation (18) is equal to characteristic equation of that (16). Therefore, the numerator of (18) is placed as follows:

$$
\begin{aligned}
& \left\{K_{d} K_{T} T_{m} R(s)+J R T_{m} I_{v}-K_{d} K_{t} T_{m} I_{p}\right\} s^{3} \\
& +\left\{\begin{array}{l}
K_{p} K_{T} T_{m} R(s)-K_{i} K_{T} T_{m} I_{i} \\
-J R \frac{T_{m}}{J}\left(-\frac{T_{L}}{J}\right)-K_{p} K_{T} T_{m} I_{p}
\end{array}\right\} s^{2} \\
& Y_{v}(s)=\frac{+\left\{K_{i} K_{T} T_{m} R(s)-K_{i} K_{T} T_{m} I_{p}\right\} s}{J R T_{m} s^{3}+\left(J R+K_{d} K_{T} T_{m}\right) s^{2}+K_{p} K_{T} T_{m} s+K_{i} K_{T} T_{m}} \\
& =\frac{s(s+\alpha)(s+\beta)}{(s+\alpha)^{3}} \\
& =\frac{s(s+\beta)}{(s+\alpha)^{2}}
\end{aligned}
$$

Furthermore, the initial value of the integrator in the PID controller $I_{i}$ is

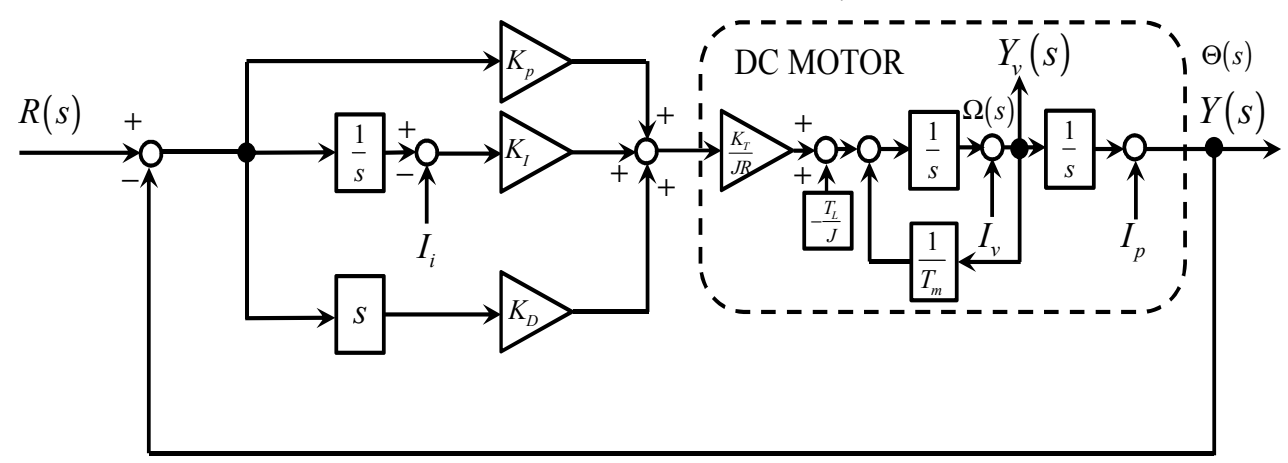

Figure 5. Block diagram of proposed method to consider initial value 


$$
\begin{gathered}
\alpha^{2}\left\{J R T_{m} I_{v}-K_{d} K_{T} T_{m} I_{p}+K_{d} K_{T} T_{m} R(s)\right\} \\
\alpha\left\{-K_{p} T_{m} K_{T} R(s)-\alpha R T_{L} T_{m}+\alpha K_{p} K_{T} T_{m} I_{p}\right\} \\
I_{i}=-\frac{+\left\{K_{i} K_{T} T_{m} R(s)-K_{i} K_{T} T_{m} I_{p}\right\}}{\alpha K_{i} K_{T} T_{m}}
\end{gathered} .
$$

Hence, the system cannot be discontinuous from the bang-bang control to the PID control.

Here, the condition of the bang-bang control to the PID control is the velocity $\omega_{f}(t)$. However, the $t_{c^{-}} \theta_{f}(t)$ graph is influenced by the changing of the torque. Therefore, we introduce the disturbance observer, and the system can be estimated as torque-disturbance. Thus, the initial value $I_{i}$ can be calculated in real-time.

\section{Simulation Study}

In this section, we show simulation results of the proposed method in several cases, which include variations of target-value, torque-disturbance, and the plant with a modelling error.

Table 1. shows each parameter value of the DC motor [5].

Table 1. DC motor parameters [5]

\begin{tabular}{ll}
\hline Parameters & Value \\
\hline$L$ & $5.6 \times 10^{-5}$ \\
$R$ & $7.4 \times 10^{-1}$ \\
$K_{E}$ & $4.06 \times 10^{-2}$ \\
$K_{T}$ & $4.15 \times 10^{2}$ \\
$J$ & $4.47 \times 10^{-1}$ \\
$D$ & 4.30 \\
$T_{L}$ & $5.5 \times 10^{2}$ \\
$u(t)$ & 12 \\
\hline
\end{tabular}

From Fig. 1 and Table 1., $Y(s)-U(s)$ and $Y(s)$-Torquedisturbance for the DC motor are written as follows:

$$
Y(s)=\theta(s)=\frac{0.0165}{s(0.0165 s+1)}(1254.6 U(s)-1230.4)
$$

The disturbance observer is written as follows:

$$
\left\{\begin{array}{c}
{\left[\begin{array}{c}
\dot{x}_{1}(t) \\
\dot{x}_{2}(t) \\
\dot{d}(t)
\end{array}\right]=\left[\begin{array}{ccc}
0 & 1 & 0 \\
0 & -60.56 & 1 \\
0 & 0 & 0
\end{array}\right]\left[\begin{array}{l}
x_{1}(t) \\
x_{2}(t) \\
d(t)
\end{array}\right]+\left[\begin{array}{l}
0 \\
1 \\
0
\end{array}\right] u(t)} \\
y(t)=\left[\begin{array}{lll}
1 & 0 & 0
\end{array}\right]\left[\begin{array}{l}
x_{1}(t) \\
x_{2}(t) \\
d(t)
\end{array}\right]
\end{array}\right.
$$

The disturbance observer gain $L_{e}$ is derived by the optimal control method, where, the weight functions $Q_{e}$ and $R_{e}$ are as follows:

$$
Q_{e}=\left[\begin{array}{ccc}
10 & 0 & 0 \\
0 & 10 & 0 \\
0 & 0 & 1 \times 10^{18}
\end{array}\right], R_{e}=1
$$

The disturbance observer gain $L_{e}$ is as follows:

$$
\boldsymbol{L}_{e}=\left[\begin{array}{lll}
1.94 \times 10^{3} & 1.88 \times 10^{6} & 1 \times 10^{8}
\end{array}\right]^{\mathrm{T}} .
$$

The reference model is as follows:

$$
\frac{150^{3}}{(s+150)^{3}}
$$

Therefore, the gains of the PID controller $K_{p}, K_{i}$, and $K_{d}$ are as follows:

$$
K_{p}=53.80 \quad K_{i}=2.69 \quad K_{d}=0.31
$$

Fig.6 shows the switching time in bang-bang input $t_{c}$ position switching signal $\theta_{f}(t)$ graph. Here, the measurement points equal 10 . The range of the measurement and the velocity switching signal $\omega_{f}(t)$ are

$$
\left\{\begin{array}{l}
t_{c}=0.01 \sim 0.1[\mathrm{sec}],(\text { increments of } 0.01) \\
\omega_{f}(t)=80
\end{array}\right.
$$

The switching of the system operates under the velocity switching signal $\omega_{f}(t)$

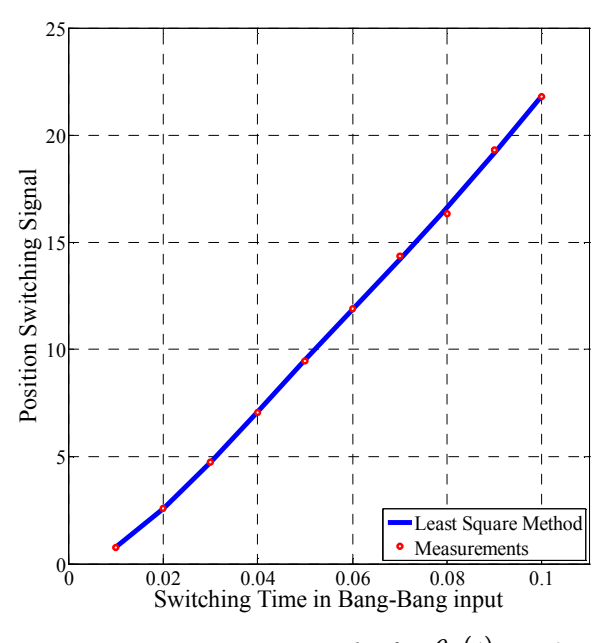

Figure 6. Measurement result of $t_{c^{-}} \theta_{f}(t)$ graph

\subsection{Variations of the Target-Value}

The simulation study in this section is performed when the target-value changes.

Here, the position switching signal $\theta_{f}(t)$ and the velocity switching signal $\omega_{f}(t)$ are as follows:

$$
\left\{\begin{array}{l}
\theta_{f}(t)=r(t)-0.8 \\
\omega_{f}(t)=80
\end{array}\right.
$$


The target values $R(s)$ are 5 and 20. Fig. 7 exhibits the responses in position and velocity.
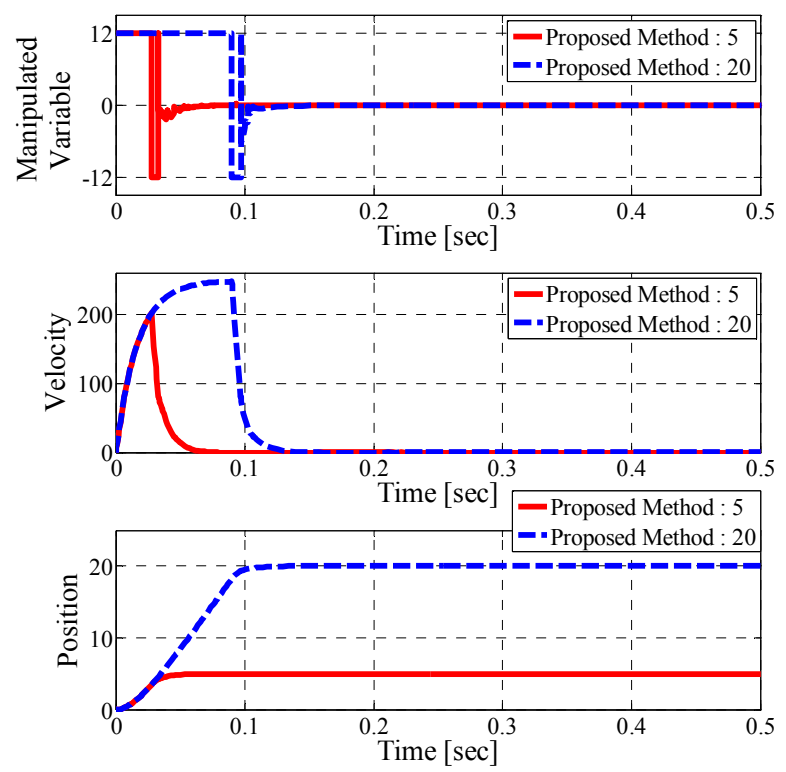

Figure 7. Responses of the target-value variation

The switching time of the bang-bang input from Fig. 6 is as follows:

$$
\left\{\begin{array} { l } 
{ R ( s ) = 5 } \\
{ R ( s ) = 2 0 }
\end{array} \Rightarrow \left\{\begin{array}{l}
t_{c}=0.028 \\
t_{c}=0.090
\end{array}\right.\right.
$$

\subsection{Variation of the Torque-Disturbance}

The simulation study in this section is performed when it changes the torque.

The target-value $R(s)$ is 20 , the position switching signal $\theta_{f}(t)$ is 19.2 and the velocity switching signal $\omega_{f}(t)$ is 80 .

To confirm the simulation results, the torque is changed as follows:

$$
-\frac{T_{L}}{J} \pm 1000
$$

Fig. 8 shows the responses in position and velocity.

\subsection{Plant with a Modeling Error}

The simulation study in this section includes the plant with a modeling error.

The target value $R(s)$ is 20 , the position switching signal $\theta_{f}(t)$ is 19.2 and the velocity switching signal $\omega_{f}(t)$ is 80 .

The plant with a modeling error from (14) is as follows:

$$
\frac{0.0165 \times( \pm 20 \%)}{0.0165 \times( \pm 20 \%) s+1}
$$

Fig. 9 shows the responses in position and velocity when the plant has a modeling error.
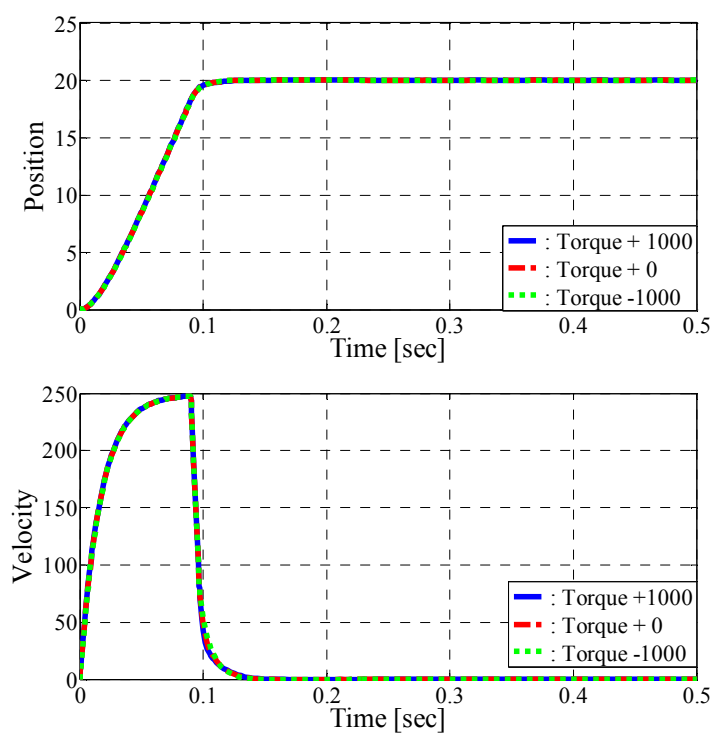

Figure 8. Responses of the torque variation

\section{Experimental Study}

This section describes the experimental results obtained using a DC motor [12] to show the effectiveness of our proposed method. In industrial applications, electric motors including DC motors are widely employed in equipment such as that used for factory automation, robotics, and hard disk drives.

The experimental control system, shown in Fig. 10, consists of a DC motor, an encoder, a counter that measures the information from an encoder, a host $\mathrm{PC}$ that transmits the control information, a target $\mathrm{PC}$ that calculates the manipulated variable, and a D/A converter.
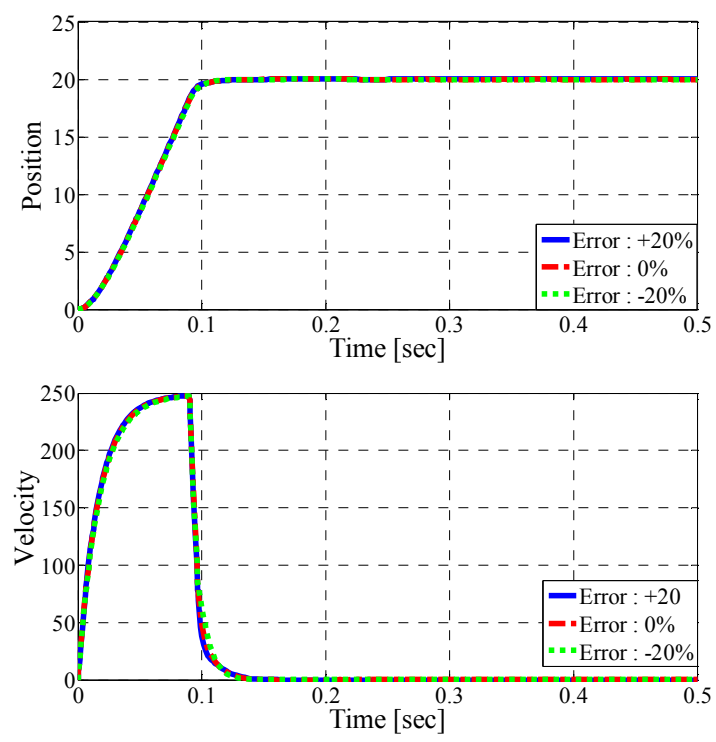

Figure 9. Responses of the plant with a modeling error

First, the control signal used to move the DC motor is transmitted from the host $\mathrm{PC}$ to the target $\mathrm{PC}$ and then to the $\mathrm{D} / \mathrm{A}$ converter with a resolution of 12 bits. Next, the output of the motor is transformed to the pulse of Phase A 
and Phase B by an encoder with a resolution of 0.0879 $\mathrm{deg} /$ count. The counter measures the pulses and transmits the positional signal to a host PC through a target PC.

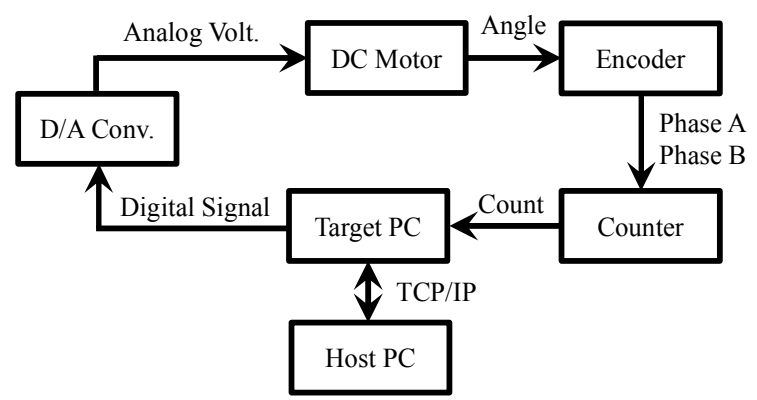

Figure 10. The experimental Control System

Fig. 11 shows the DC motor used in the experimental study, which was developed by the PID Corporation (PID-QET ii). The motor is equipped with an inertial load disk that has the radius of $0.0248 \mathrm{~m}$ and a weight of 0.068 $\mathrm{kg}$.

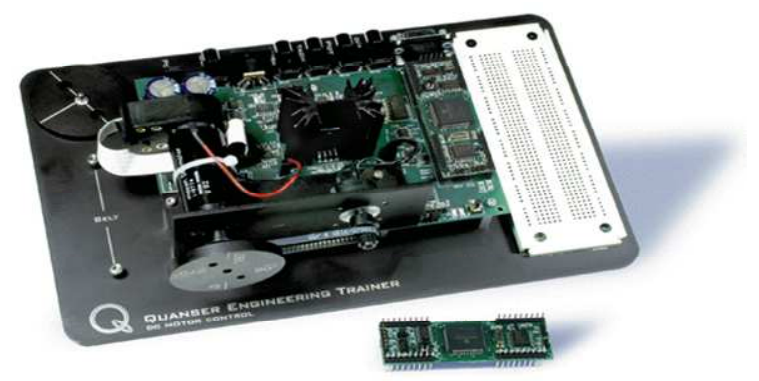

Figure 11. The DC motor

The transfer function of the DC motor is as follows:

$$
\frac{339.6}{s(s+10.78)}
$$

The disturbance observer is written as follows:

$$
\left\{\begin{array}{c}
{\left[\begin{array}{c}
\dot{x}_{1}(t) \\
\dot{x}_{2}(t) \\
\dot{d}(t)
\end{array}\right]=\left[\begin{array}{ccc}
0 & 1 & 0 \\
0 & -10.78 & 1 \\
0 & 0 & 0
\end{array}\right]\left[\begin{array}{c}
x_{1}(t) \\
x_{2}(t) \\
d(t)
\end{array}\right]+\left[\begin{array}{l}
0 \\
1 \\
0
\end{array}\right] u(t)} \\
y(t)=\left[\begin{array}{lll}
1 & 0 & 0
\end{array}\right]\left[\begin{array}{l}
x_{1}(t) \\
x_{2}(t) \\
d(t)
\end{array}\right]
\end{array}\right.
$$

The disturbance observer gain $L_{e}$ is derived by the optimal control method, where the weight functions $Q_{e}$ and $R_{e}$ are as follows:

$$
Q_{e}=\left[\begin{array}{ccc}
1 \times 10^{2} & 0 & 0 \\
0 & 1 \times 10^{5} & 0 \\
0 & 0 & 1 \times 10^{18}
\end{array}\right], R_{e}=1
$$

$$
L_{e}=\left[\begin{array}{lll}
1.00 \times 10^{3} & 1.93 \times 10^{5} & 3.16 \times 10^{7}
\end{array}\right]^{T}
$$

The reference model is as follows:

$$
\frac{90^{3}}{(s+90)^{3}}
$$

The gains of the PID controller $K_{p}, K_{i}$, and $K_{d}$ are as follows:

$$
K_{p}=71.55 \quad K_{i}=214.6 \quad K_{d}=0.76
$$

Fig.12 shows the switching time in the bang-bang input $t_{c}$-position switching signal $\theta_{f}(t)$ graph. Here, the measurement points equal 10 . The ranges of the measurement and the velocity switching signal $\omega_{f}(t)$ are as follows:

$$
\left\{\begin{array}{l}
t_{c}=0.01 \sim 0.1[\mathrm{sec}],(\text { increments of } 0.01) \\
\omega_{f}(t)=30
\end{array}\right.
$$

The switching of the system operates under the velocity switching signal $\omega_{f}(t)$

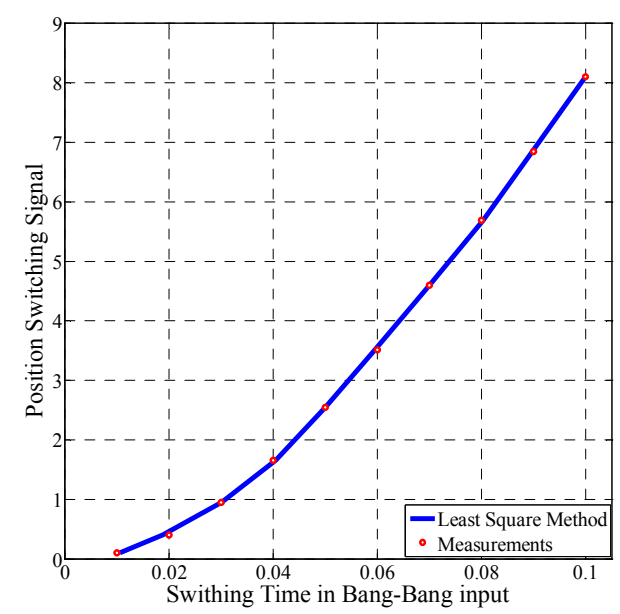

Figure 12. Measurement result of $t_{c^{-}} \theta_{f}(t)$ graph

\subsection{Variations of the Target-Value}

In this experimental study, the performance is shown when changing the target-value.

Here, the position switching signal $\theta_{f}(t)$ and the velocity switching signal $\omega_{f}(t)$ are as follows:

$$
\left\{\begin{array}{l}
\theta_{f}(t)=r(t)-0.5 \\
\omega_{f}(t)=30
\end{array}\right.
$$

The target-values $R(s)$ are 2 and 8. Fig. 13 exhibits the responses in position and velocity. The switching time of the bang-bang input from Fig. 12 is as follows:

The disturbance observer gain $L_{e}$ is as follows: 


$$
\left\{\begin{array} { l } 
{ R ( s ) = 2 } \\
{ R ( s ) = 8 }
\end{array} \Rightarrow \left\{\begin{array}{l}
t_{c}=0.0385 \\
t_{c}=0.0948
\end{array}\right.\right.
$$

\subsection{Confirmation of the Robustness}

The study in this section shows the robustness of the proposed method.

The target value $R(s)$ is 8 , the position switching signal $\theta_{f}(t)$ is 7.5 and velocity switching signal $\omega_{f}(t)$ is 30 .

Fig. 14 shows the responses in position and velocity when a plant has a weight or a belt in which weight has a radius of $0.0248 \mathrm{~m}$ and a weight of $0.001 \mathrm{~kg}$.
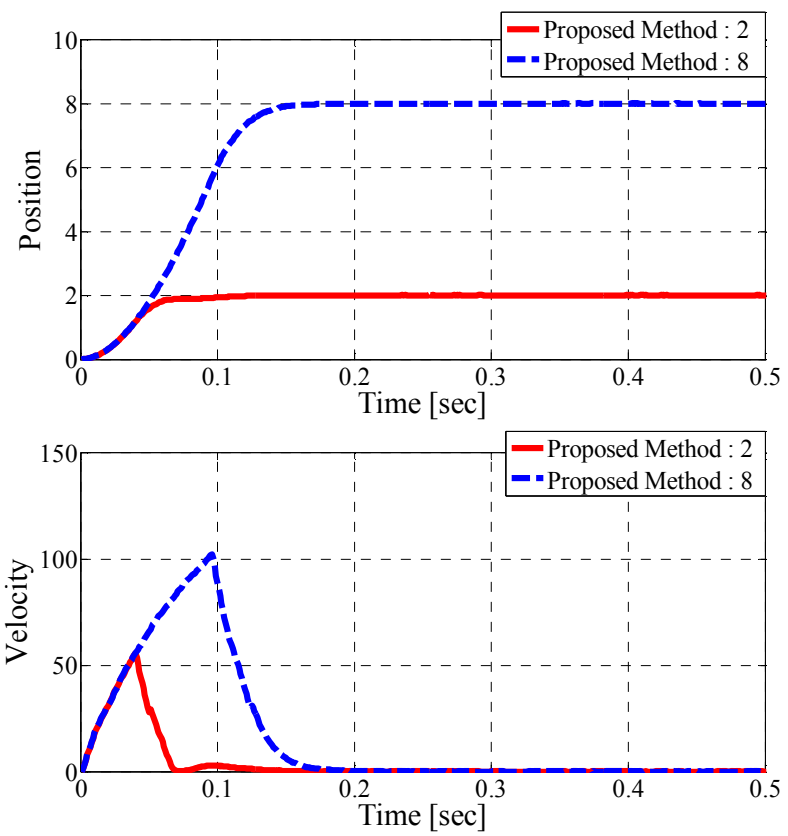

Figure 13. The responses of the torque variation
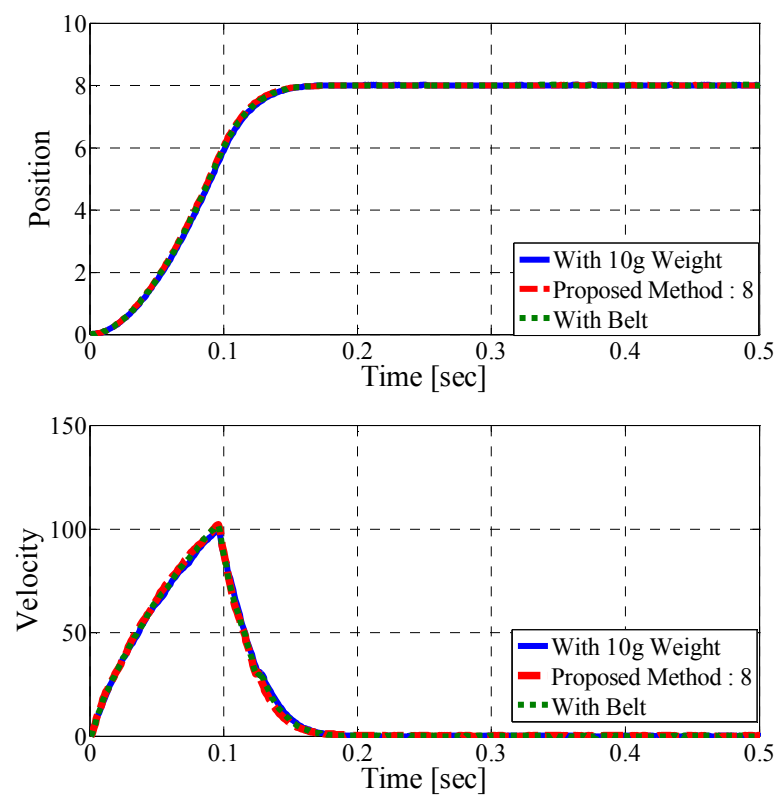

Figure 14. The responses of the torque variation

\section{Conclusion}

This study proposed a positioning control with high-speed starting and stopping for a DC motor using bang-bang control.

It is easy to determine the switching time of the bang-bang input from graph measurement and theoretical calculations.

When the control system is switched from "Bang-Bang control" to "PID control", it becomes a discontinuous system without the initial value of the integrator of the PID controller. Therefore, we introduced an initial value for the system which is calculated by theoretical analysis. In addition, we obtained the initial value of the integrator in real time by introducing a disturbance observer. Therefore, the system became resistant to changes in torque.

In the simulation and experimental studies, we confirmed the effectiveness of the proposed method in various cases. The responses of the position and the velocity can be obtained when changing cases caused by variations of the target-value, torque-disturbances, and the plant with a modeling error. We can get superior performances in various cases in these studies.

\section{References}

[1] N. Suda, System Control and information Society, PID control, Asakura Publication Company , 1992 (In Japanese)

[2] S. Yamamoto, and N. Kato, PID control Foundation and Application, 2nd ed., Asakura Publication Company, 2005 (In Japanese)

[3] S. Miyazaki, and J. Miyazaki, Application of automatic control learning on computer, , CQ Publication Company, 1991 (In Japanese)

[4] J.P. Lasalle, "The 'Bang-Bang' Principle", Automatic and remote control, Vol.1, Proc. of the First International Congress of the International Federation of Automatic Control, pp.493-497., 1960

[5] K. Nagakura, "Time-Optimal Precise Positioning Control using a DC Servomotor", Journal of The Japan Society for Precision Engineering, Vol. 43, No.9, pp.11056-1062. Sept. 1977(In Japanese)

[6] James. H , Rillings , and Rob J.Roy, "Bang-Bang Control Strategy Using a Tabular Adaptive Model", J IEEE Trans. Automatic Control,Vol.12, Issue 3, pp.310-312, Jun.1967.

[7] K. Furuta, S. Kobayashi, "Bang-Bang Position of Direct Drive Motor", Proc. of IECON'90, 16th Annual Conference of IEEE, Industrial Electronics Society,pp.148-153, Nov. 1990

[8] N. Koreta, H. Okitomo, K. Tsumura, K.Takeuchi, and T.Egawa, "Study of High Accuracy of Machine Tool with Bang-Bang Control", Journal of The Japan Society for Precision Engineering, Vol.6, No.3, pp.427-431, Sept. 1977 (in Japanese)

[9] M. G.Vasek, and R.T. O'Brien. Jr., "Bang-Bang Control of Double Integrator", Proc. The Thirty-Forth Southeastern Symposium on System Theory 2002, pp.275-278, Mar. 2002 
[10] Y. Yoshida, and T. Sogo, "Real Time Bang Bang Control of Crane", Departmental Bulletin Paper, Pub. Of Institute of Science and Technology Research, Chubu Univ. Vol.22, 6 page on PDF, Sept. 2010 (in Japanese)

[11] H. Shibasaki, R. Tanaka, H. Ogawa, Y Ishida, "High Speed Activation and Stopping Control System Using the Bang-Bang Control for a DC Motor", Proc. of 2013 IEEE International Symposium on Industrial Electronics (ISIE2013), pp.1-6, May, 2013.
[12] R. Tanaka, H. Shibasaki, H. Ogawa, T. Murakami, and Y. Ishida, "Controller design approach based on linear programming", ISA Trans., vol. 52, pp. 744-751, Nov., 2013. 